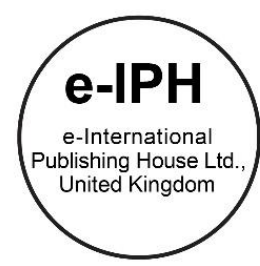

\title{
Postnatal Confinement among Malay Women: Comparing practices between two different generations
}

\author{
Bahiyah Abdullah¹,2, Suzanna Daud ${ }^{1,2}$, Mariam Mohamad³, Akmal Z M Zahid² \\ ${ }^{1}$ Maternofetal and Embryo (MatE) Research Group, ${ }^{2}$ Department of Obstetrics and Gynaecology, ${ }^{3}$ Department of Population Health \& \\ Preventive Medicine, Faculty of Medicine, \\ Universiti Teknologi MARA (UiTM), 47000 Selangor, Malaysia
}

bahiyah@uitm.edu.my, suzannadaud@hotmail.com, mariammd@uitm.edu.my, akmalmohdzahid@yahoo.com Tel:+60192205150

\begin{abstract}
This study aimed to determine the difference in postnatal confinement practice between two different generations. It was an unmatched cross-sectional study involving 200 women. Women aged less than 50 , and 50 years old or more, were grouped as younger and older generation, respectively. This study showed there were significant differences in postnatal confinement practice including the pattern of food restriction and some traditional postnatal care practices (less sexual abstinence, hot compression, herbal bath and avoiding social outing among women from younger generations). Clinically proven intervention should be recommended, and harmful practices should be discouraged to ensure a safe confinement practice.
\end{abstract}

Keywords: post-partum; puerperium; traditional; era

eISSN: 2398-4287 @ 2019. The Authors. Published for AMER ABRA cE-Bs by e-International Publishing House, Ltd., UK. This is an open access article under the CC BYNC-ND license (http://creativecommons. org/licenses/by-nc-nd/4.0). Peer-review under responsibility of AMER (Association of Malaysian Environment-Behaviour Researchers), ABRA (Association of Behavioural Researchers on Asians) and cE-Bs (Centre for Environment-Behaviour Studies), Faculty of Architecture, Planning \& Surveying, Universiti Teknologi MARA, Malaysia.

DOI: https://doi.org/10.21834/e-bpj.v4i12.1911

\subsection{Introduction}

The postpartum period is an interval when a woman's body gradually regain back its non-pregnant state physiologically and anatomically. Confinement means the state of being restricted, and there is no standardisation of definition about its use as a confinement practice postnatally. Generally, confinement is a prescribed set of rules of dos and don'ts during the immediate postnatal period. The duration of practice varies between different regions of the world. The postpartum confinement is uncommon in the Western countries, but it is a deeply rooted practice within the Asian culture, including among Malay women. The continued practice is despite the global changes taking place within the social, economic and education of the younger generation. In Singapore, up to $96 \%$ of women still adhere to traditional confinement practice (Fok et al., 2016), and the confinement practice remains prevalent among Asian women (Withers, Kharazmi, \& Lim, 2018). Despite lack of evidence on its effects on women's postnatal health, it continues to be practised due to multiple presumed advantages to the postpartum mothers and their newborn babies such as faster recovery, preventing from future joint ache and various illnesses (Naser et al., 2012; Withers et al., 2018).

Even though the confinement practice may differ between ethnicities, there are similarities in practice which usually include food restrictions and recommendations, behavioural restrictions and the use of traditional herbs and massage (Fadzil, Shamsuddin, \& Wan Puteh, 2016). With the advance of scientific evidence and healthcare services, we need to review the confinement practice, which has been passed down through generations to ensure that it is safe and beneficial to the mother.

eISSN: 2398-4287 @ 2019. The Authors. Published for AMER ABRA cE-Bs by e-International Publishing House, Ltd., UK. This is an open access article under the CC BYNC-ND license (http://creativecommons.org/licenses/by-nc-nd/4.0/). Peer-review under responsibility of AMER (Association of Malaysian Environment-Behaviour Researchers), ABRA (Association of Behavioural Researchers on Asians) and cE-Bs (Centre for Environment-Behaviour Studies), Faculty of Architecture, Planning \& Surveying, Universiti Teknologi MARA, Malaysia.

DOI: https://doi.org/10.21834/e-bpj.v4i12.1911 
Some research has suggested that adherence to confinement practice is declining and there is also concern on adverse effects associated with confinement, such as postpartum depression and venous thromboembolism. However, there was no study looking at current women's practice, in comparison with women from the earlier generation, particularly among Malay women population. Therefore, there is a need to establish possible changes in confinement practice and whether the changes are beneficial or more detrimental to women's health. A healthcare provider needs to know and understand this issue to allow effective postnatal care while respecting individual woman's cultural belief. Hence, we aim to study the differences in confinement practice between women from the older and younger generation.

\subsection{Literature Review}

Traditional postnatal confinement has been practised over generations among women, particularly in many Asian countries (Withers et al., 2018). Despite differences in the way postpartum confinement was practised in different ethnicities and cultures, it shares similar objectives and purpose. The main aim of postnatal confinement is mainly to facilitate the mother recuperating after a pregnancy and delivery because women are considered to be weak, fragile and vulnerable to illness. It also meant to curtail the risk of getting any infections to the mother and her baby during this recovery period (Withers et al., 2018).

Various literature documented similar postnatal confinement concern that in failing to comply with the traditional postpartum confinement practice, this may result in multiple future negative health implications. It includes earlier ageing, increased risk of various chronic ailments, joint aches and body aches (Davis, 2001; Fadzil et al., 2016; Withers et al., 2018). Therefore, many women continue to practise postnatal confinement despite lack scientific explanation partly due to concern on potential complications that may arise and many women practising it out of respect for the older women who are taking care of them during this postnatal period (Sheehy, Aung, \& Foster, 2016).

The concept of 'heat' and 'cold' is the primary influence of postnatal confinement practice. The belief that mothers lose their body heat during childbirth, which leads to an imbalance between 'heat' and 'cold' forces in women's bodies (Withers et al., 2018). It explains why most confinement practice entails similar themes: rest and getting the balance of 'heat' and 'cold' in the body (Davis, 2001; KimGodwin, 2003; Naser et al., 2012). It also has become the basis of most food and behavioural recommendation and restriction of the postnatal confinement practice. However, some literature has addressed the concern about some potentially harmful practices in the traditional confinement practise (Ngunyulu, Mulaudzi, \& Peu, 2015).

The details on methods of the postnatal confinement vary considerably across cultures, but most are related to food and behavioural recommendation and restriction (Withers et al., 2018). A study among Malaysian women previously showed that most Malaysian women, although from different ethnic groups, share similarities in their postnatal confinement practice. For example, they had many similar food taboos, received traditional postpartum massage, consumed traditional herbs, and the older female family members are often actively involved in caring them during this postpartum period (Fadzil et al., 2016).

On the other hand, a study in Singapore demonstrated some significant difference in the postnatal confinement practice between different ethnic groups. They found that Chinese mothers showered less and were more likely to depend on confinement nannies during their confinement period than Malay and Indian mothers. They also found that significantly more Malay mothers used confinement massage therapy than the other two ethnic groups (Fok et al., 2016).

Many literatures have described on the various postnatal confinement practise in many different population and cultures(Fadzil et al., 2016; Lundberg \& Trieu, 2011; Naser et al., 2012; Piperata, 2008; Raven, Chen, Tolhurst, \& Garner, 2007; Sharma, van Teijlingen, Hundley, Angell, \& Simkhada, 2016; Sheehy et al., 2016; Shrestha, 2014). Therefore, it is crucial for the healthcare providers who run the postnatal care service to incorporate and shared a culturally appropriate advice, to improve women's compliance and ultimately to achieve the best outcome for both postpartum mother and their babies (Fantaye, Gunawardena, \& Yaya, 2019; Kim-Godwin, 2003; Leung, Arthur, \& Martinson, 2005; Lundberg \& Trieu, 2011; Sein, 2013).

In most cultures, the concept of heat imbalances found in the body affects the dietary prescription during postnatal confinement. The recommendation of 'hot' foods and drinks while avoiding 'cold' foods and beverages is to promote the circulation of energy in the body (Withers et al., 2018). This concept also influences various behavioural recommendations such as the use of blanket, sweaters, socks and different herbal preparation, to keep the body heat. In particular culture, use of steam placed atop beds with fire and medicinal leaves burning underneath and the practice of hot compress are among ways recommended to restore lost heat (Withers et al., 2018). Confinement massages are also routinely practised across many cultures. However, with a case reported on a woman sustained a cervical vertebra fracture causing her being tetraparesis (Sahathevan et al., 2011) should raise a concern of potential harm that this may cause, particularly in the hand of inexperienced accoucheur. Another confinement taboo is the recommendation for sexual abstinence throughout the confinement period. It has been part of the confinement practice in many cultures including China, Thailand, Vietnam, Malaysia, Turkey and Myanmar (Geckil, Sahin, \& Ege, 2009; Withers et al., 2018).

\subsection{Methodology}

It was a cross-sectional study, conducted at a neighbourhood in Pasir Mas, Kelantan in November 2014. It was mainly populated by Malays and following similar Malay culture, including postnatal confinement practice. There were about 500 living quarters in the area, which was our sampling frame. We used a random number generator to select the living quarters, and we approached all female occupants who resided in the selected living-quarters that fulfilled our inclusion criteria to be our respondents. The inclusion criteria were Malay women who had a history of at least one birth, no previous history of mental illness and consented to participate in our study. 
Participants were grouped into either the younger generation (any women who aged less than 50 years old) and the older generation (any women who aged 50 years old or more).

The sample size required in this study was calculated using StatCalc in Epi Info software. We used sample size calculation for unmatched cross-sectional study design with $80 \%$ power and $95 \%$ two-sided confidence level, with the prevalence of $96 \%$ women practised confinement routines (Fok et al., 2016) and to detect 15\% difference, plus 10\% non-response, we required approximately 100 respondents per group.

A structured questionnaire consisted of demographic details, background on her confinement practices, dietary pattern (avoided food and commonly taken food) during confinement and various standard practices during confinement were used during a face-to-face interview with participants by trained interviewers. For women who had more than one delivery, we obtained the details on their confinement practice after their first delivery. The list of foods and practices enquired in this questionnaire were based on what has been reported in the previous literature (Fok et al., 2016) and standard practices known to the authors.

Data were analysed using statistical software (IBM SPSS version 22). Pearson chi-square and Fisher exact tests were used to detecting statistically significant differences in confinement practices between young and old generations. The significant level is taken at a $p$-value less than 0.05 .

This study has been approved by the Universiti Teknologi MARA Research Ethics Committee on 22nd July 2014 (Reference number: 600-RMI (5/1/6)).

\subsection{Results}

A total of 200 respondent participated in this study, in which 99 women were from the younger generation, and 101 women were from the older generation. The mean age of the younger generation group was 28.4 (SD 4.1) years old, and the mean age for the older population was 66.1 (SD 8.1) years old. The median parity for the younger group was 2 (range 1-6) and 5 (range 1-13) for the older group.

There were no enquiries made on the educational status, average family income and occupation because this study compared women in two different eras. Therefore, the socioeconomic, access to education and the general community situation were more likely to be different between the different generations.

Table 1 showed the comparison of the background of confinement practice between these two groups. It showed there was a significant difference in place of confinement and accompanying person during confinement between the two groups.

Table 1: Detail background of our respondent

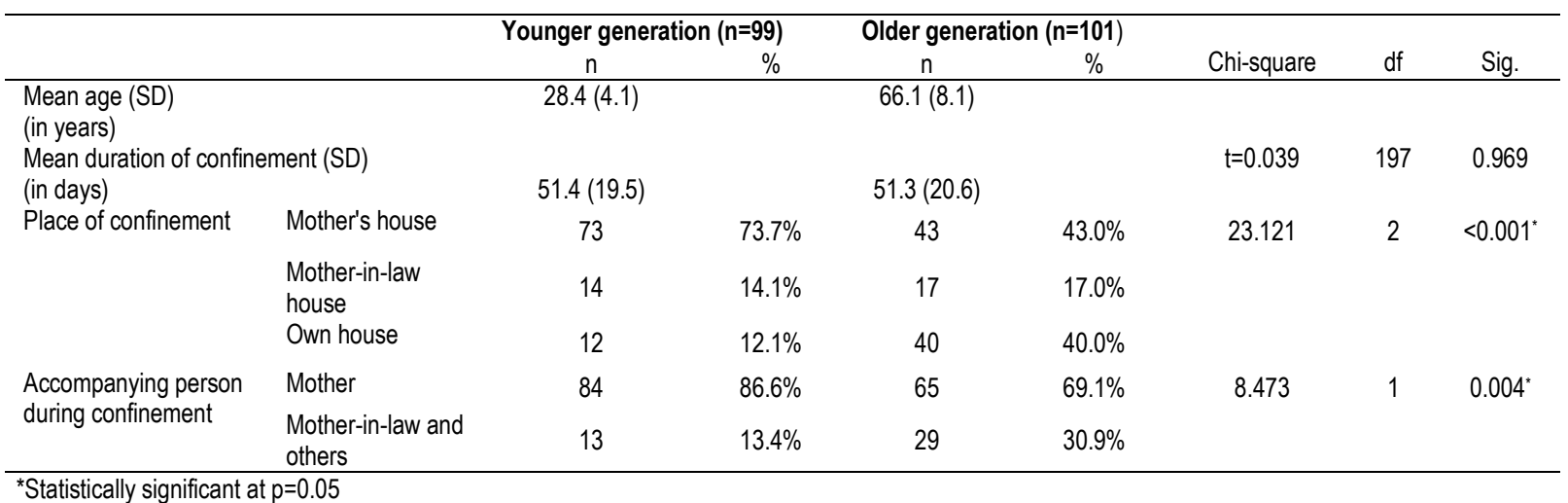

Figure 1 and Figure 2 demonstrated the dietary pattern during confinement between these two groups. Figure 1 showed that fewer women from younger generation restricted their intake of egg, milk and green vegetables compared to the older generation. Figure 2 demonstrated that fewer women in the younger generation took 'Morinda citrifolia' and tonic from medicinal plants compared to the older generation. There were fewer women in the younger generation group who practised restriction of fluid intake compared to the older generation. 


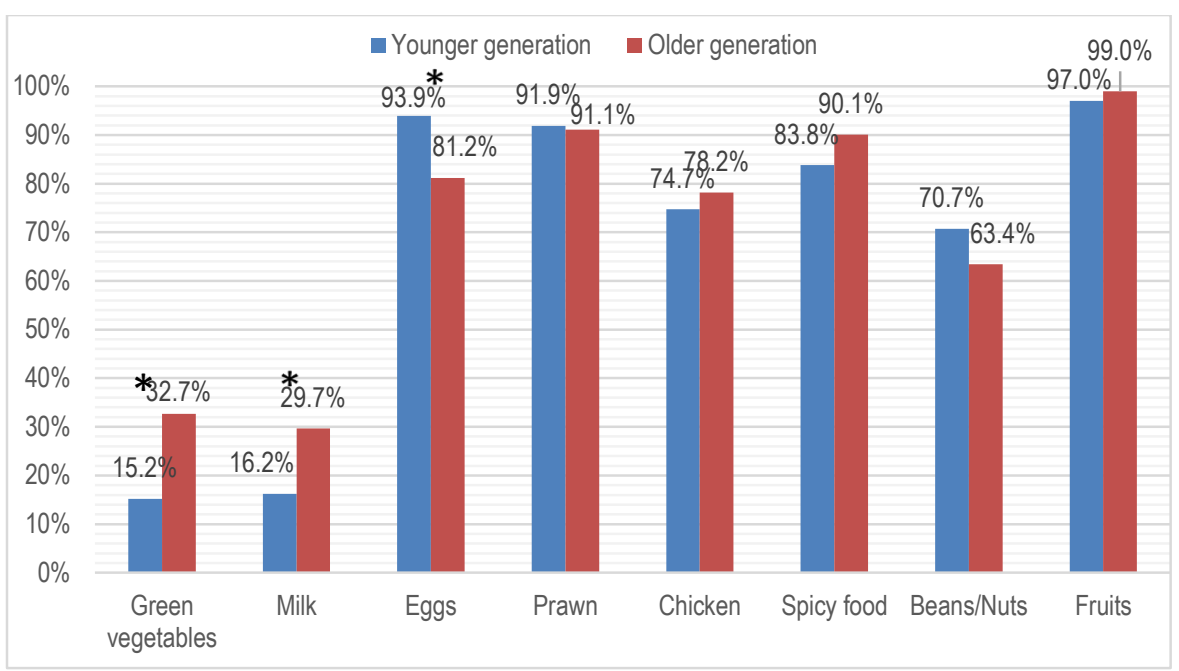

*Statistically significant at $p=0.05$

Fig 1: Food restrictions during confinement

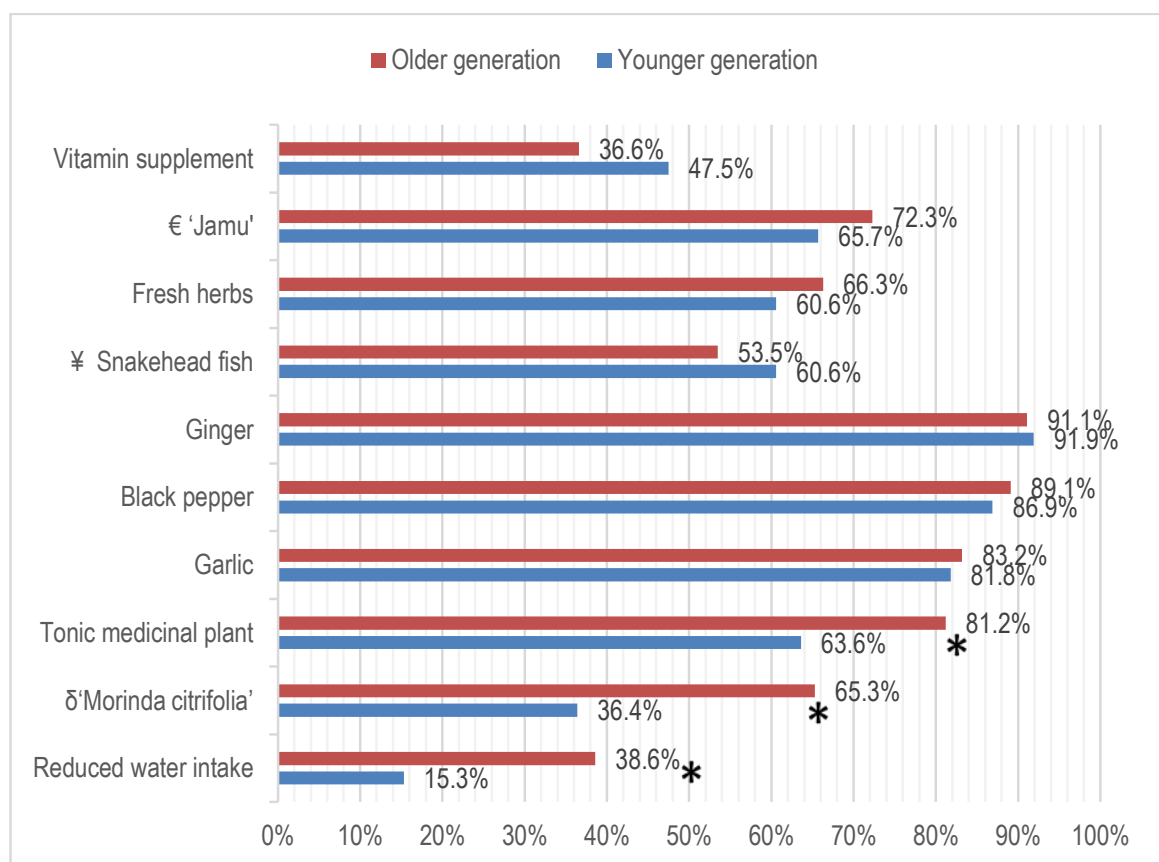

*Statistically significant at $\mathrm{p}=0.05$

$\delta$ 'Morinda citrifolia' is a plant called as 'mengkudu' in Malay.

¥'Snakehead fish' is called as 'ikan haruan' in Malay.

$€$ 'Jamu' is a traditional medicine made predominantly herbal medicine and made from the natural product.

Fig 2: Dietary recommendation during confinement

Apart from dietary practice, we also surveyed on the differences in other behavioural practices during confinement, as demonstrated in Figure 3. Significantly more women from the older generation practised hot compression, applying 'param' and taking a herbal bath compared to the younger generation. On top of that, significantly more women from the older generation comply with the recommendation for sexual abstinence during confinement and avoiding social outing during their confinement period compared to younger generations. 


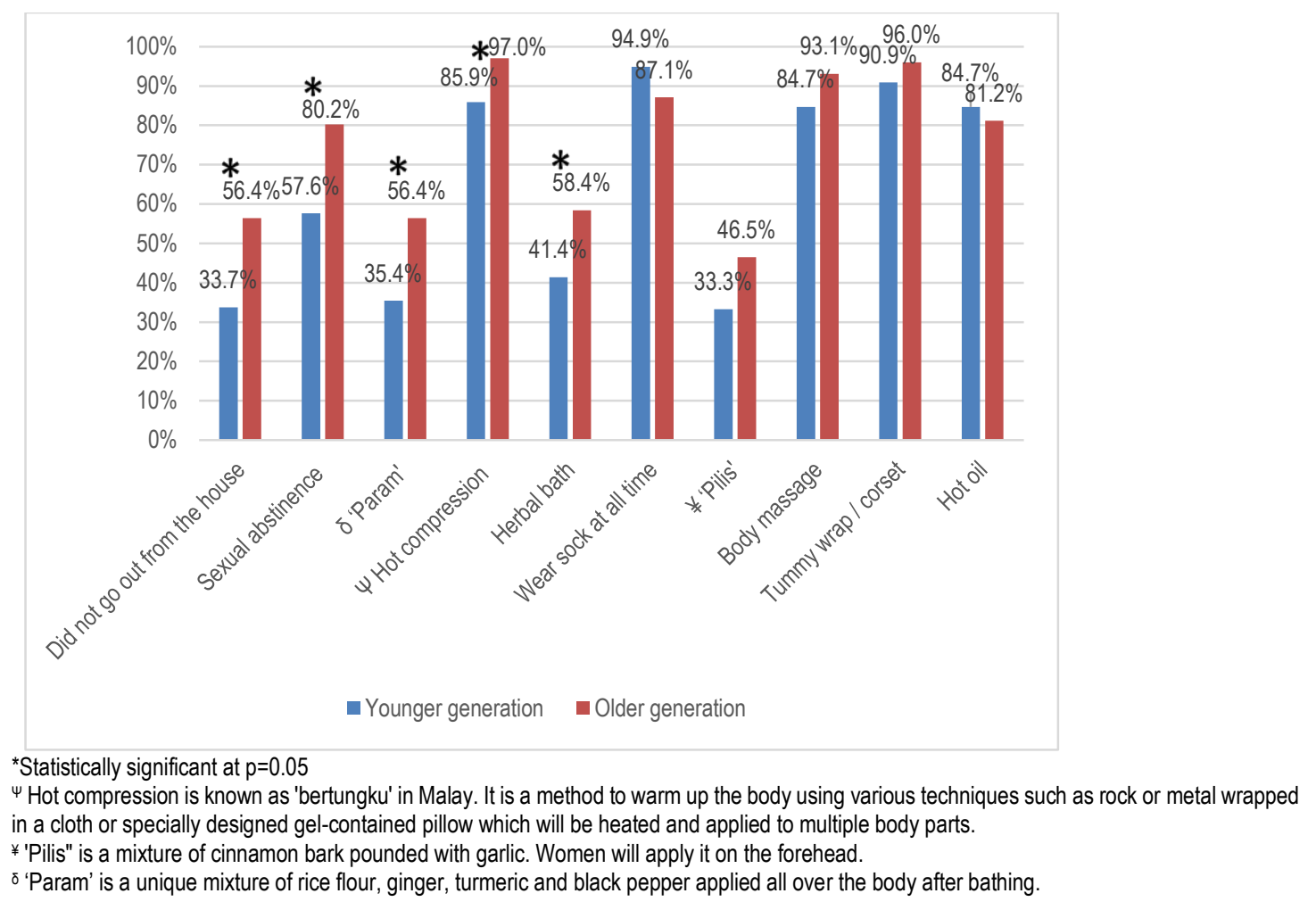

Figure 3: Common behavioural confinement practices among Malay women

\subsection{Discussion}

This study found that the majority of the Malay women from the younger generation still practise the traditional confinement with few differences and modifications identified. This study demonstrated that the younger generation preferred to have their confinement period with their mother. Having someone who is trusted, reliable and caring during the confinement period is essential to reduce the new mother's stress level after delivery, especially in addition to coping with the newborn baby. Confinement practice itself aims to reduce the incidence of postpartum depression (Wong \& Fisher, 2009). A systematic review of its efficacy is conflicting. However, a supportive environment and any factors that can reduce the mother's stress are known to be significant protective factors against postpartum depression (Özcan, Boyacıoğlu, \& Dinç, 2017; Shi, Ren, Li, \& Dai, 2018). Hence, women should be allowed and even encouraged to choose with whom they wish to be with during the confinement period.

Nutritional recommendation and restriction are one of the essential components in confinement practice (Fok et al., 2016; Lamxay, de Boer, \& Bjork, 2011; Naser et al., 2012; Shrestha, 2014). Firstly, with regards to food restriction during confinement, this study found that significantly fewer women from the younger generation follow the restriction in consuming green vegetables and milk. Even though there was a minority of women from both generations comply with this restriction, a further significant reduction in women following this restriction indicates a positive change because of these are a good source of vitamins and fibres.

This study found that the majority of women from both generations avoid taking eggs during confinement. However, surprisingly, there were significantly more women from the younger generation practise restriction in consuming eggs during confinement than women from the older generation. This study also demonstrated that the majority of women from the younger generation still follow the restriction of taking chicken and prawn. Avoiding eggs, chicken and prawn were due to the belief that they can cause a delay in wound healing, and they can cause itchiness over the wound. Therefore, healthcare providers are responsible for educating women and clearing this misunderstanding because, in the absence of allergy, these foods are good sources of protein and iron for the mother.

On top of that, the majority of women from younger generation remained to avoid fruits during confinement because of it was believed to be a 'cold' food and not suitable for the general health of postnatal mothers. Sadly, this restriction deprives them of the benefits of fruits which are rich in vitamins, minerals and fibres. Nuts and spicy food are another type of food restriction practised. Despite lack of evidence, nuts, soybean and spicy food, have been associated with producing excessive wind which may cause colic to the baby. Hence this restriction may be reasonable.

Next is on food recommendation during confinement. In Malay culture, one of the advices is for women to reduce water intake because culturally, the belief is it will cause a delay in wound healing and slower recovery during the postnatal period. However, fluid restriction during confinement is a potentially harmful practice. Due to the physiological changes that take place during pregnancy, the postpartum period remains a hypercoagulable state. The hypercoagulability in combination with the effect of dehydration due to fluid restriction and being physically less active especially those who have undergone operative deliveries will further increase their risk to develop venous thromboembolism (D'Alton et al., 2016). It is one of the essential yet preventable causes of maternal death (Kevane et 
al., 2014; Parunov, Soshitova, Ovanesov, Panteleev, \& Serebriyskiy, 2015). Therefore, it is imperative to advise women to ensure adequate hydration antenatally until after deliveries. It is also vital to ensure good breastmilk production. Therefore, a significant reduction in the number of women from the younger generation following this advice is a positive change which could be due to increasing awareness as a result of health education given during pregnancy-care and after-delivery care offered by the healthcare provider.

There are other several commonly recommended foods during confinement. This includes ginger, black pepper, 'Morinda citrifolia', garlic, snakehead fish and 'jamu'. Majority of women from both generation groups did follow this recommendation. However, 'Morinda citrifolia' or known among Malays as 'mengkudu' and tonic from medicinal plants were significantly less consumed by women from the younger generation. This study did not explore the reason for this however it is probably due to the increasing trend of women taking various prepared herbal products or in a 'jamu' form which is available in many herbal shops and more consumer-friendly. It is also more challenging to obtain the traditional herbs in its original form and more challenging to prepare. There is a wide range of herbal products aimed at postnatal women in the market, which combines various types of herbs hence making comparison difficult.

Nevertheless, it is of concern because they lack data on safety and efficacy. To our knowledge, there is so far no published data regarding the transfer of these various herbal products through the breastmilk. Hence any short-term or long-term adverse effects to the baby are unknown. The use of traditional herbal medicines product has been addressed over the years globally. It has led to a set of legislation and regulations (Serafini, Stanzione, Foddai, Anton, \& Delmulle, 2012; Silano, Coppens, Larranaga-Guetaria, Minghetti, \& Roth-Ehrang, 2011) which varies between regions, mainly to protect the consumers. Unfortunately, the compliance towards those regulations and its enforcement needs to be improved.

In a nutshell, food recommendations and restrictions during the confinement period should be allowed as long as it is safe and beneficial according to the evidenced-based nutritional guidance. Harmful pieces of advice, such as restriction of fluid, must be discarded. Balanced and nutritious food intake will contribute to a speedy recovery of the mother and successful breastfeeding for a healthy baby.

With regards of the behavioural recommendation and restriction during confinement, there were significantly fewer women in a younger generation observe some traditional confinement practices such as hot compression, applying 'param' and taking a herbal bath. These practices tend to be more time consuming and often there is increasing difficulty in finding assistance to perform these rituals. Therefore, these practices are slowly becoming out of favour amongst younger women. Significantly fewer women from younger generation comply with the restriction from social outing from the house during confinement. The restrictions were in place with the good intention of giving time to the mother to recover, rest from her usual hectic chores (e.g. shopping, sending children to school etc.) and to facilitate mother and child bonding. Although it potentially intended to serve as protection and privilege, it may not be suitable for all women. For some women, this restriction may cause undue stress and lead to increased risk of postpartum depression.

Apart from that, postnatal women were usually advised to refrain from having sexual intercourse until the lochia has ceased, as part of health and religious advice. However, prolonging the duration of abstinence throughout the whole confinement period is usually a cultural practice. This study found that fewer younger women observed this restriction. Hence many of them will resume the intimate relationship with their partner earlier, while still in the confinement period. Currently, there is no published literature on advantageous or disadvantageous of prolonging the sexual abstinence throughout the confinement period.

The limitation of this study is there is no validated questionnaire available for assessing confinement practice. Therefore, this questionnaire was created based on the literature on confinement practice and what was known to the authors.

\subsection{Conclusion}

Postnatal confinement period, which is a common practice within the Asian culture, may continue to be practised, guided by evidencebased medicine. Traditional confinement practice should be seen as a privilege for the mother. Hence mothers should be allowed to practise according to guidance from the healthcare provider. Clinically proven intervention such as pelvic floor exercise, back care, healthy eating and regular exercises should come hand-in-hand during confinement. Harmful practice such as fluid restriction should be discouraged, together with the explanation of the danger of such practice. Healthcare providers should avoid belittling or dismissing confinement practice, as this may deter the relationship between women and healthcare providers. A diplomatic approach is required to ensure a safe confinement practice for the mother and the newborn baby.

\section{Acknowledgements}

The LESTARI Research Grant (Universiti Teknologi MARA) has funded this study. (Reference number: 600-IRMI/DANA 5/3/LESTARI (0177/2016)

\section{References}

D'Alton, M. E., Friedman, A. M., Smiley, R. M., Montgomery, D. M., Paidas, M. J., D'Oria, R., ... Clark, S. L. (2016). National Partnership for Maternal Safety: Consensus Bundle on Venous Thromboembolism. Anesth Analg, 123(4), 942-949. doi:10.1213/ane.0000000000001569 
Fadzil, F., Shamsuddin, K., \& Wan Puteh, S. E. (2016). Traditional Postpartum Practices Among Malaysian Mothers: A Review. J Altern Complement Med, 22(7), 503508. DOI:10.1089/acm.2013.0469

Fantaye, A. W., Gunawardena, N., \& Yaya, S. (2019). Preferences for formal and traditional sources of childbirth and postnatal care among women in rural Africa: A systematic review. PLoS One, 14(9), e0222110. DOI:10.1371/journal.pone.0222110

Fok, D., Aris, I. M., Ho, J., Lim, S. B., Chua, M. C., Pang, W. W., Chong, Y. S. (2016). A Comparison of Practices During the Confinement Period among Chinese, Malay, and Indian Mothers in Singapore. Birth, 43(3), 247-254. DOl:10.1111/birt.12233

Geckil, E., Sahin, T., \& Ege, E. (2009). Traditional postpartum practices of women and infants and the factors influencing such practices in South-Eastern Turkey. Midwifery, 25(1), 62-71. DOl:10.1016/j.midw.2006.12.007

Kevane, B., Donnelly, J., D'Alton, M., Cooley, S., Preston, R. J., \& Ni Ainle, F. (2014). Risk factors for pregnancy-associated venous thromboembolism: a review. J Perinat Med, 42(4), 417-425. DOI:10.1515/jpm-2013-0207

Kim-Godwin, Y. S. (2003). Postpartum beliefs and practices among non-Western cultures. MCN Am J Matern Child Nurs, 28(2), 74-78; quiz 79-80.

Lamxay, V., de Boer, H. J., \& Bjork, L. (2011). Traditions and plant use during pregnancy, childbirth and postpartum recovery by the Kry ethnic group in Lao PDR. J Ethnobiol Ethnomed, 7, 14. DOI:10.1186/1746-4269-7-14

Leung, S. K., Arthur, D., \& Martinson, I. M. (2005). Perceived stress and support of the Chinese postpartum ritual "doing the month". Health Care Women Int, $26(3), 212$ 224. DOI:10.1080/07399330590917771

Lundberg, P. C., \& Trieu, T. N. (2011). Vietnamese women's cultural beliefs and practices related to the postpartum period. Midwifery, 27(5), $731-736$. DOI:10.1016/j.midw.2010.02.006

Naser, E., Mackey, S., Arthur, D., Klainin-Yobas, P., Chen, H., \& Creedy, D. K. (2012). An exploratory study of traditional birthing practices of Chinese, Malay and Indian women in Singapore. Midwifery, 28(6), e865-871. DOl:10.1016/j.midw.2011.10.003

Ngunyulu, R. N., Mulaudzi, F. M., \& Peu, M. D. (2015). Comparison between indigenous and Western postnatal care practices in Mopani District, Limpopo Province, South Africa. Curationis, 38(1). DOl:10.4102/curationis.v38i1.1252

Özcan, N. K., Boyacıoğlu, N. E., \& Dinç, H. (2017). Postpartum Depression Prevalence and Risk Factors in Turkey: A Systematic Review and Meta-Analysis. Archives of Psychiatric Nursing, 31(4), 420-428. doi:https://doi.org/10.1016/j.apnu.2017.04.006

Parunov, L. A., Soshitova, N. P., Ovanesov, M. V., Panteleev, M. A., \& Serebriyskiy, II. (2015). Epidemiology of venous thromboembolism (VTE) associated with pregnancy. Birth Defects Res C Embryo Today, 105(3), 167-184. DOI:10.1002/bdrc.21105

Piperata, B. A. (2008). Forty days and forty nights: a biocultural perspective on postpartum practices in the Amazon. Soc Sci Med, 67(7), 1094-1103. DOI:10.1016/j.socscimed.2008.05.031

Raven, J. H., Chen, Q., Tolhurst, R. J., \& Garner, P. (2007). Traditional beliefs and practices in the postpartum period in Fujian Province, China: a qualitative study. BMC Pregnancy Childbirth, 7, 8. DOI:10.1186/1471-2393-7-8

Sahathevan, R., Tan, H. J., Abdullah, S., Shahizon, A. M., Hamidon, B. B., \& Raymond, A. A. (2011). Spinal cord compression following traditional confinement massage. Med J Malaysia, 66(5), 495-496. Retrieved from https://www.ncbi.nlm.nih.gov/pubmed/22390109

Sein, K. K. (2013). Beliefs and practices surrounding postpartum period among Myanmar women. Midwifery, 29(11), 1257-1263. DOI:10.1016/j.midw.2012.11.012

Serafini, M., Stanzione, A., Foddai, S., Anton, R., \& Delmulle, L. (2012). The European role on traditional herbal medicinal products and traditional plant food supplements. J Clin Gastroenterol, 46 Suppl, S93-94. DOI:10.1097/MCG.0b013e318266b08f

Sharma, S., van Teijlingen, E., Hundley, V., Angell, C., \& Simkhada, P. (2016). Dirty and 40 days in the wilderness: Eliciting childbirth and postnatal cultural practices and beliefs in Nepal. BMC Pregnancy Childbirth, 16(1), 147. DOI:10.1186/s12884-016-0938-4

Sheehy, G., Aung, Y., \& Foster, A. M. (2016). "She Learned it from her Mother and Grandmother": Women's Experiences with Delivery and Post-partum Practices in Periurban Yangon, Myanmar. Matern Child Health J, 20(4), 854-861. DOl:10.1007/s10995-016-1918-z

Shi, P., Ren, H., Li, H., \& Dai, Q. (2018). Maternal depression and suicide at immediate prenatal and early postpartum periods and psychosocial risk factors. Psychiatry Res, 261, 298-306. DOI:10.1016/j.psychres.2017.12.085

Shrestha, K. (2014). Food practices among postnatal mothers in a hilly township in Northeastern Nepal. Nepal Med Coll J, 16(2-4), 135-138.

Silano, V., Coppens, P., Larranaga-Guetaria, A., Minghetti, P., \& Roth-Ehrang, R. (2011). Regulations applicable to plant food supplements and related products in the European Union. Food Funct, 2(12), 710-719. DOI:10.1039/c1fo10105f

Withers, M., Kharazmi, N., \& Lim, E. (2018). Traditional beliefs and practices in pregnancy, childbirth and postpartum: A review of the evidence from Asian countries Midwifery, 56, 158-170. DOl:10.1016/j.midw.2017.10.019

Wong, J., \& Fisher, J. (2009). The role of traditional confinement practices in determining postpartum depression in women in Chinese cultures: a systematic review of the English language evidence. J Affect Disord, 116(3), 161-169. DOI:10.1016/j.jad.2008.11.002 